\title{
Genetic Variation in Glutamate Carboxypeptidase II and Interaction with Dietary Natural Vitamin C May Predict Risk for Adenomatous Polyp Occurrence
}

\author{
Jeong-Hwa Choi ${ }^{1,4}$, Zoe Yates ${ }^{2}$, Charlotte Martin ${ }^{1}$, Lyndell Boyd ${ }^{1}$, Xiaowei Ng ${ }^{1}$, \\ Virginia Skinner ${ }^{3}$, Ron Wai ${ }^{3}$, Jeongseon Kim ${ }^{4}$, Hae Dong Woo ${ }^{4}$, Martin Veysey ${ }^{3}$, \\ Mark Lucock ${ }^{1 *}$
}

\begin{abstract}
Background: The C1561T variant of the glutamate carboxypeptidase II (GCPII) gene is critical for natural methylfolylpolyglutamte (methylfolate) absorption, and has been associated with perturbations in folate metabolism and disease susceptibility. However, little is known on C1561T-GCPII as a risk factor for colorectal cancer. Therefore, this study examined whether C1561 T-GCPII influences folate metabolism and adenomatous polyp occurrence. Materials and Methods: 164 controls and 38 adenomatous polyp cases were analysed to determine blood folate and plasma homocysteine (Hcy) level, dietary intake of natural methylfolate, synthetic pteroylglutamic acid (PteGlu), vitamin C and C1561 T-GCPII genotype. Results: In controls and cases, 7.3 and 18.4 percent of subjects respectively, were found to have the CT genotype, increasing the risk for adenomatous polyp occurrence 2.86 times $(95 \% \mathrm{CI}: 1.37-8.0, \mathrm{p}=\mathbf{0 . 0 3 5}$ ). Total dietary folate, methylfolate and PteGlu intake and the level of erythrocyte folate and plasma Hcy did not predict the occurrence of an adenomatous polyp. However, dietary natural vitamin $C$ intake was associated with adenomatous polyp risk within C1561T-GCPII CT genotype subjects ( $\mathrm{p}=\mathbf{0 . 0 3 7}$ ). Conclusions: The findings suggest that $\mathrm{C1561T-GCPII}$ variation may be associated with risk for adenomatous polyp, and vitamin $\mathrm{C}$ may modify risk by interacting with the variant gene, its expression product and/or folate substrates.
\end{abstract}

Keywords: Adenomatous polyp - colorectal cancer - folate - GCPII - polymorphism - vitamin C

Asian Pac J Cancer Prev, 16 (10), 4383-4386

\section{Introduction}

Folate, a water soluble B vitamin, is the generic term for both natural folylpolyglutamate derivatives (including methylfolate), and synthetic folic acid (pterolymonoglutamic acid, PteGlu). Folate consists of three molecular components; a pteridine ring, paraaminobenzoic acid and glutamic acid residues conjugated to the pteridine (Lucock, 2000). The natural form of folate found in food generally has five to eight glutamic acid residues, but only folylmonoglutamic acid can be absorbed across the membrane of enterocytes. For this reason, polyglutamate forms of methylfolate require glutamate carboxypeptidase II (GCPII) to hydrolyse (deconjugate) the glutamic acid residues prior to absorption (Chandler et al., 1986).

GCPII, a Zn-dependant exopeptidase, is a type II transmembrane glycoprotein locates on the brush-border of the intestine. GCPII and its genetic variants are known to be associated with folate nutritional status and disease susceptibility, as this is a critical locus for the absorption mechanism of methylfolate (Devlin et al., 2000). One particular mutation of this gene, $\mathrm{C} 1561 \mathrm{~T}$ in which histidine 475 is changed to tyrosine (rs61886492, H475Y), has been reported to be associated with risk for breast and prostate cancer, coronary artery disease and miscarriage (Divyya et al., 2012; Vijaya Lakshmi et al., 2013). This genetic variant may lead to a reduction in GCPII activity, and hence to a lower blood folate concentration and higher homocysteine (Hcy), potentially implicating this polymorphism in the pathoaetiology of these disorders (Devlin et al., 2000).

Folate has long been considered a significant nutrient in the aetiology of cancer, consistent with its biochemical properties in cellular metabolism. Diminished intake of dietary folate and a reduced blood status may lead to alterations in major biosynthetic pathways involving folate as a one-carbon carrier (Liu et al., 2011). These potentially include altered S-adenosylmethionine, purine and thymidilate synthesis. Perturbations in these pathways can result in multiple molecular effects which contribute

${ }^{1}$ School of Environmental and Life Sciences, ${ }^{2}$ Biomedical Sciences and Pharmacy, University of Newcastle, ${ }^{3}$ Teaching \& Research Unit, Central Coast Local Health District, Australia, ${ }^{4}$ Molecular Epidemiology Branch, National Cancer Center, , Ilsandong-gu, Goyang-si Gyeonggi-do, Korea*For correspondence: mark.lucock@newcastle.edu.au 
to the development and progression of cancer (Jing et al., 2012; Gao et al., 2013). Epidemiological data provides clear evidence that folate plays a critical role in colorectal cancer occurrence either on its own or in concert with folate genetic variants (Roswall et al., 2010; Kim et al., 2012; Liu et al., 2013), although little work exists on C1561T-GCPII as a risk factor for colorectal cancer via alteration to folate metabolism.

This study examined the potential influence of C1561T-GCPII on folate metabolism and the consequent susceptibility for adenomatous polyp occurrence, an antecedent of colorectal cancer. Erythrocyte folate and plasma Hcy level, and dietary folate (natural methyl folate and synthetic PteGlu) were determined. Additionally, vitamin $\mathrm{C}$ intake which is critical for helping to maintain the redox status and bioavailability of methylfolate vitamers ( $\mathrm{Ng}$ et al., 2008) was also examined in this context.

\section{Materials and Methods}

\section{Subjects}

Two hundred and two participants were recruited from a local gastroenterology clinic (Gosford, NSW, Australia). Participants were aged between 40 and 89 years, and mean age for all subjects was 63.2 years. All subjects joined in the study voluntarily, and informed consent was obtained prior to entering the study. Ethics approval for this research was given by the local Human Research Ethics Committee prior to the commencement of the research (H-429-0407). As a result of colonoscopy, 38 participants (18.8\%) were diagnosed with an adenomatous polyp (cases), while controls were defined as participants who had no evidence of an adenomatous polyp during colonoscopy. There was no difference for distribution of sex and age by adenomatous polyp phenotype.

\section{Sample collection and folate, homocysteine and genotype} analysis

In order to determine C1561T-GCPII genetic variation and erythrocyte folate and plasma Hcy level, $5 \mathrm{~mL}$ of whole blood was collected by venipuncture in an EDTA Vacuette. Erythrocyte folate was determined using a chemiluminescent immunoassay (Access Immunoassay System, Bechman Instruments, Inc). The determination of Hcy was performed using HPLC with fluorescence detection. The Hcy assay used for this study was modified from the methods of Araki and Sako (Araki and Sako, 1987). The C1561T-GCPII genotype was measured according to Lucock et al. (2013).

Food frequency questionnaire for determination of dietary folate and vitamin $C$ intake

An individual interviewer-administered food frequency questionnaire (FFQ) was conducted on the day of examination. The FFQ covered 225 types of food items generally consumed in Australia, additionally all participants were requested to provide a list of supplements they were taking. Nutrient intake analysis from the FFQ was performed using Foodworks ${ }^{\mathrm{TM}} 2.10 .146$ (Xyris Software, Brisbane, QLD, Australia). Methylfolate and synthetic PteGlu intake were determined, respectively. This involved discrimination between natural methylfolate and the artificial PteGlu of the vitamin supplements and fortified food. Similarly, vitamin $\mathrm{C}$ was estimated separately according to its source. All blood samples and FFQ data were collected prior to the commencement of mandatory folate fortification $\left(19^{\text {th }}\right.$ September, 2009 in Australia).

\section{Statistical Analysis}

All statistical analysis was performed using the SAS program for Windows (version 9.3; SAS Institute Inc., Cary, NC, USA). Chi-square analysis was used to determine the Hardy-Weinberg equilibrium. A t-test was used to assess the differences in mean values for each variable between categories. A logistic regression analysis was used to establish a prediction model for adenomatous polyp susceptibility with the numbers from the presence or absence of the polymorphism in cases and controls. All statistical analysis was performed using a confidence level of $95 \%$.

\section{Results}

Table 1 presents the distribution of C1561T-GCPII genotypes in the study population. The homozygous recessive genotype (TT) did not exist in either the case or the control subjects, and minor allele frequency was $4 \%$ and $9 \%$ in each group, respectively. Twelve controls and seven cases were found to have the CT genotype, and analysis gave an odds ratio (OR) of 2.86 [95\% confidence interval (CI): 1.04-7.85]. When age and sex were taken into account, again, the C1561T-GCPII variant increased the risk for adenomatous polyp (OR: 2.88. 95\% CI:

Table 1. Distribution of C1451T-GCPII Genotype and its Association with Adenomatous Polyp Occurrence

\begin{tabular}{lccc}
\hline & Control (\%) & $\begin{array}{c}\text { Adenomatous } \\
\text { Polyp (\%) }\end{array}$ & OR* (95\% CI) \\
\hline CC & $152(92.7)$ & $31(81.6)$ & Reference \\
CT & $12(7.3)$ & $7(18.4)$ & $2.88(1.37-8.0)$ \\
TT & 0 & 0 & - \\
MAF & 0.04 & 0.09 & - \\
Total & $164(100)$ & $38(100)$ & - \\
\hline
\end{tabular}

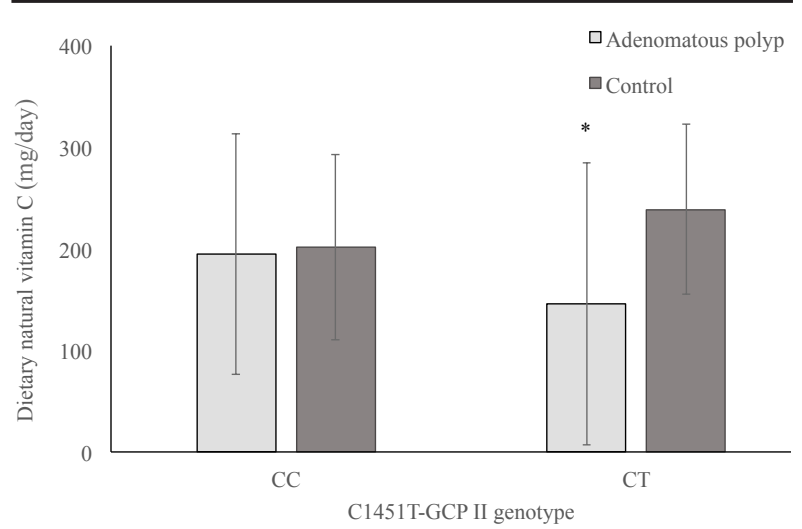

Figure 1. A Comparison of Dietary Vitamin C Intake by C1451T-GCPII Genotype and Adenomatous Polyp Phenotype; *denotes significance with a confidence level of $95 \%$ 

1.37-8.0).

Dietary folate intake (total dietary folate, methylfolate and PteGlu) and the level of erythrocyte folate and plasma Hcy were not associated with the occurrence of adenomatous polyp either dependent or independent of C1561T-GCPII genotype (data not shown). However, within the C1561T-GCPII CT genotype group, subjects with adenomatous polyp and controls showed a significant difference in their dietary natural vitamin $C$ intake ( $\mathrm{p}=0.037$, Figure 1). In individuals with CT genotype and adenomatous polyps, the level of dietary natural vitamin $\mathrm{C}$ was $145.6 \pm 91.4 \mathrm{mg} / \mathrm{day}$, which was significantly lower than those of CT subjects without an adenomatous polyp (238.8 $\pm 83.7 \mathrm{mg} /$ day). This may suggest that dietary natural vitamin $\mathrm{C}$ intake is associated with adenomatous polyp risk within the C1561T-GCPII CT genotype group.

\section{Discussion}

Colonocytes are known to exhibit a high demand for folate. This stems from short lifespan (3-5days) but fast regeneration rate (Potten and Allen, 1977), making folate a critical nutrient in colonic pathoaetiology. This is particularly true in the case of cancer, since folate is a major supplier of one-carbon groups for purine and thymidylate synthesis and methylation.

The findings from the present study suggest that the C1561T-GCPII variant, which is central to natural methylfolate absorption and subsequent metabolism may be associated with adenomatous polyp occurrence. The presence of the polymorphic allele of C1561T-GCPII may increase the risk for an adenomatous polyp, while dietary vitamin $\mathrm{C}$ intake may modify that risk, interacting with the variant gene.

GCPII is required to deconjugate glutamic acid residues from the folate molecule since only folylmonoglutamic acid can be absorbed. The C1561T-GCPII variation in exon 13 of the catalytic domain of this gene results in a histidine to tyrosine transition at position 475 , which results in altered ligand binding, hence, interfering with GCPII activity (Devlin et al., 2000; Mesters et al., 2006). Impaired intestinal absorption of folate due to the genetic variant may therefore alter folate metabolism with consequences for disease susceptibility. Although C1561T-GCPII variation did not show a significant difference for blood folate and Hcy levels by adenomatous polyp phenotype in the present study, a number of other reports suggest C1561T-GCPII variation affects folate metabolism and linking it to disease. Divyya et al. (2012) reported that C1561T-GCPII variation was associated with risk for coronary artery disease and miscarriage, but only had an influence on folate metabolism in subjects taking the lowest tertile of dietary folate. The difference in plasma folate level by $1561 \mathrm{~T}$ allele was not evident in the subjects with higher dietary folate intake. The mean folate intake level $(454.5 \mu \mathrm{g} / \mathrm{day})$ in the current study is relatively higher than in other populations in Europeans $(225 \mu \mathrm{g} /$ day), North Americans (352 $\mu \mathrm{g} /$ day and $422 \mu \mathrm{g} /$ day) and other Australian cohorts (314 $\mu \mathrm{g} /$ day) (Clapin et al., 2012). The high intake of folate in the current subjects, possibly due to discretionary use of PteGlu, would not require substantial GCPII activity since the synthetic PteGlu is already in monoglutamate form. In fact, the abundance of dietary PteGlu may nullify the influence derived from C1561T-GCPII on folate metabolism (Divyya et al., 2012).

Vitamin $\mathrm{C}$ is a key factor in the stabilizing and salvaging of labile natural methylfolyl vitamers, and likely modifies the erythrocyte folate level on its own, or in concert with other folate genetic variations (Lucock et al., 2013). In this study, within the CT genotype subjects, dietary natural vitamin $\mathrm{C}$ intake may be associated with adenomatous polyp occurrence. Adenomatous polyp patients with the CT genotype had significantly lower dietary natural vitamin $C$ intake $(145.6 \pm 91.4 \mathrm{mg} /$ day $)$ than normal controls $(238.8 \pm 83.7 \mathrm{mg} / \mathrm{day}, \mathrm{p}=0.037)$. Since vitamin $\mathrm{C}$ is a strong antioxidant, higher intake of dietary natural vitamin $\mathrm{C}$ may influence the integrity of the expression products of variant genes by modifying redox status (Lucock et al., 2013). In addition, higher vitamin $\mathrm{C}$ intake may also help to maintain the reduced status of the methylfolate vitamers, and hence improve the bioavailability of dietary folate ( $\mathrm{Ng}$ et al., 2008). The $\mathrm{C} 1561 \mathrm{~T}$ heterozygote encodes a protein which shows altered activity/expression, and hence may affect folate absorption and nutritional status, compared to wildtype. However increased bioavailability of dietary methylfolate with vitamin $\mathrm{C}$ may reduce risk for adenomatous polyp when GCPII is encoded by the heterozygous GCPII genotype.

It is interesting to note that this association between dietary natural vitamin C and C1561T CT genotype was not found for total dietary vitamin $\mathrm{C}$ intake (including both natural and synthetic form). A small number of studies have reported a differential role for nutrients in disease aetiology, particularly in relation to their physicochemical properties. For instance, dietary methylfolate is generally protective against colorectal cancer, whereas the intake of synthetic PteGlu does not show such a positive effect, or may actually be associated with risk for colorectal cancer (Cole et al., 2007; Roswall et al., 2010). Additionally, the protective effect of "natural vitamin C" may actually come from unknown compounds, not examined in the current study, which are recognised as present in "healthy" foods that are typically rich in other vitamins, minerals and antioxidant phytoprotectants-a typical limitation of many nutritional studies.

The results from the present study are interesting given that the C1561T-GCPII variant is associated with risk for adenomatous polyp occurrence and that vitamin $\mathrm{C}$ may modify the risk by interacting with the GCPII variants. However, the results shown here are derived from a relatively small cohort. Despite this, the novel findings surrounding the C1561T-GCPII variant and dietary vitamin $\mathrm{C}$ in adenomatous polyp aetiology are clearly interesting and should be verified with further studies.

\section{References}

Araki A, Sako Y (1987). Determination of free and total homocysteine in human plasma by high-performance liquid chromatography with fluorescence detection. J Chromatogr, 422, 43-52. 
Chandler CJ, Wang TT, Halsted CH (1986). Pteroylpolyglutamate hydrolase from human jejunal brush borders. Purification and characterization. J Biol Chem, 261, 928-33.

Clapin HF, Fritschi L, Iacopetta B, et al (2012). Dietary and supplemental folate and the risk of left- and right-sided colorectal cancer. Nutr Cancer, 64, 937-45.

Cole BF, Baron JA, Sandler RS, et al (2007). Folic acid for the prevention of colorectal adenomas: a randomized clinical trial. JAMA, 297, 2351-9.

Devlin AM, Ling EH, Peerson JM, et al (2000). Glutamate carboxypeptidase II: a polymorphism associated with lower levels of serum folate and hyperhomocysteinemia. Hum Mol Genet, 9, 2837-44.

Divyya S, Naushad SM, Addlagatta A, et al (2012). Paradoxical role of C1561T glutamate carboxypeptidase II (GCPII) genetic polymorphism in altering disease susceptibility. Gene, 497, 273-9.

Gao S, Ding LH, Wang JW, et al (2013). Diet folate, DNA methylation and polymorphisms in methylenetetrahydrofolate reductase in association with the susceptibility to gastric cancer. Asian Pac J Cancer Prev, 14, 299-302.

Jing C, Huang Z, Duan Y, et al (2012). Folate intake, methylenetetrahydrofolate reductase polymorphisms in association with the prognosis of esophageal squamous cell carcinoma. Asian Pac J Cancer Prev, 13, 647-51.

Kim J, Cho YA, Kim DH, et al (2012). Dietary intake of folate and alcohol, MTHFR C677T polymorphism, and colorectal cancer risk in Korea. Am J Clin Nutr, 95, 405-12.

Liu AY, Scherer D, Poole E, et al (2013). Gene-diet-interactions in folate-mediated one-carbon metabolism modify colon cancer risk. Mol Nutr Food Res, 57, 721-34.

Liu YX, Wang B, Wan MH, et al (2011). Meta-analysis of the relationship between the metholenetetrahydrofolate reductase C677T genetic polymorphism, folate intake and esophageal cancer. Asian Pac J Cancer Prev, 12, 247-52.

Lucock M (2000). Folic acid: nutritional biochemistry, molecular biology, and role in disease processes. Mol Genet Metab, 71, 121-38.

Lucock M, Yates Z, Boyd L, et al (2013). Vitamin C-related nutrient-nutrient and nutrient-gene interactions that modify folate status. Eur J Nutr, 52, 569-82.

Mesters JR, Barinka C, Li W, et al (2006). Structure of glutamate carboxypeptidase II, a drug target in neuronal damage and prostate cancer. EMBO J, 25, 1375-84.

Ng X, Lucock M, Veysey M (2008). Physicochemical effect of $\mathrm{pH}$ and antioxidants on mono-and triglutamate forms of 5-methyltetrahydrofolate, and evaluation of vitamin stability in human gastric juice: Implications for folate bioavailability. Food Chemistry, 106, 200-10.

Potten CS, Allen TD (1977). Ultrastructure of cell loss in intestinal mucosa. J Ultrastruct Res, 60, 272-7.

Roswall N, Olsen A, Christensen J, et al (2010). Micronutrient intake and risk of colon and rectal cancer in a Danish cohort. Cancer Epidemiol, 34, 40-6.

Vijaya Lakshmi SV, Naushad SM, Seshagiri Rao D, et al (2013). Oxidative stress is associated with genetic polymorphisms in one-carbon metabolism in coronary artery disease. Cell Biochem Biophys, 67, 353-61. 